\title{
Inhibition of CCL19 benefits non-alcoholic fatty liver disease by inhibiting TLR4/NF-кB-p65 signaling
}

\author{
JIAJING ZHAO $^{1 *}$, YINGJUE WANG $^{1 *}$, XI WU $^{2}$, PING TONG $^{3}$, YAOHAN YUE ${ }^{1}$, \\ SHURONG GAO ${ }^{1}$, DONGPING HUANG ${ }^{4}$ and JIANWEI HUANG ${ }^{4}$
}

${ }^{1}$ Department of Traditional Chinese Medicine, Putuo District People's Hospital of Shanghai City, Shanghai 200060;

${ }^{2}$ Department of Endocrinology, Huashan Hospital, Fu Dan University, Shanghai 200040;

Departments of ${ }^{3}$ Endocrinology and ${ }^{4}$ General Surgery, Putuo District People's Hospital of Shanghai City,

Shanghai 200060, P.R. China

Received January 26, 2018; Accepted August 21, 2018

DOI: $10.3892 / \mathrm{mmr} .2018 .9490$

\begin{abstract}
Non-alcoholic fatty liver disease (NAFLD), which affects approximately one-third of the general population, has become a global health problem. Thus, more effective treatments for NAFLD are urgently required. In the present study, high levels of C-C motif ligand 19 (CCL19), signaling pathways such as Toll-like receptor 4 (TLR4)/nuclear factor- $\mathrm{KB}$ (NF- $\mathrm{\kappa B})$, and proinflammatory factors including interleukin-6 (IL-6) and tumor necrosis factor- $\alpha$ (TNF- $\alpha$ ) were detected in NAFLD patients, thereby indicating that there may be an association between CCL19 and these factors in NAFLD progression. Using a high-fat diet (HFD), the present study generated a Sprague-Dawley rat model of NAFLD, which displayed dyslipidemia with increased levels of plasma aspartate aminotransferase, alanine aminotransferase, total cholesterol and triglyceride. Dyslipidemia, liver histopathology and gene expression analyses indicated that the NAFLD model was successfully induced by HFD, and metformin and berberine (BBR) were effective treatments for NAFLD. HFD-induced CCL19 levels and associated factors were markedly reduced by the two drug treatments. In addition, metformin or BBR alone significantly promoted adenosine monophosphate-activated protein kinase (AMPK) phosphorylation, which was inhibited by HFD. These results demonstrated
\end{abstract}

Correspondence to: Dr Jiajing Zhao, Department of Traditional Chinese Medicine, Putuo District People's Hospital of Shanghai City, 1291 Jiangning Road, Putuo, Shanghai 200060, P.R. China

E-mail: zhaojiajingrose@126.com

Dr Jianwei Huang, Department of General Surgery, Putuo District People's Hospital of Shanghai City, 1291 Jiangning Road, Putuo, Shanghai 200060, P.R. China

E-mail: huangjianwei1124@live.cn

${ }^{*}$ Contributed equally

Key words: non-alcoholic fatty liver disease, C-C motif ligand 19, berberine, phospho-adenosine monophosphate-activated protein kinase, Toll-like receptor 4/nuclear factor- $\mathrm{\kappa B}-\mathrm{p} 65$, metformin that metformin and BBR could improve NAFLD, which may be via the activation of AMPK signaling, and the high expression of CCL19 in NAFLD was significantly reduced by metformin and BBR. It could be inferred that inhibition of CCL19 may be an effective treatment for NAFLD.

\section{Introduction}

Fatty liver diseases, whose prevalence is continuously rising worldwide, especially in developed countries, are characterized by excessive hepatic fat accumulation (1). Various causes, including a high-fat/calorie diet, alcoholism and other metabolic disorders, can lead to fatty liver diseases. According to different causes, fatty liver can be divided into alcoholic fatty liver disease and non-alcoholic fatty liver disease (NAFLD) (2). NAFLD, covering a spectrum of chronic liver diseases, including simple fatty liver, fatty hepatitis, fibrosis, cirrhosis, and hepatic carcinoma, affects approximately $20-40 \%$ of the general population (3). NAFLD is considered to be the hepatic manifestation of metabolic dysfunction and is strongly related to obesity, insulin resistance (IR), and dyslipidemia $(4,5)$.

Studies have shown that the accumulation of fat in hepatocytes mainly comprises triglyceride (TG) deposition (6). Diet, de novo synthesis and adipose tissue are three major sources of the acquisition of hepatic TG. Likewise, total cholesterol (TC) is related to the severity of NAFLD (7). In addition, it has been reported that increased concentrations of alanine aminotransferase (AST) and alanine aminotransferase (ALT) in serum commonly lead to NAFLD (8). Sterol response element-binding protein (SREBP)-1c, which is primarily present in the liver, is negatively regulated by AMP-activated protein kinase (AMPK) (9) and usually regulates lipogenesis as a transcription factor binding to target lipogenic genes, such as fatty acid synthase (FAS) $(10,11)$. Furthermore, SPEBP-1c has been suggested to stimulate the synthesis of TG with the synergistic effect of ChREBP (12).

Toll-like receptor 4 (TLR4), the most characteristic member of the TLR family, commonly functions as a pattern-recognition receptor. Studies have closely related TLR4 to the pathogenesis of NAFLD, and it has further been shown that NAFLD may be prevented by allelic variants of TLR4 in humans (13). Additionally, it is well-known that 
the nuclear factor (NF)- $\mathrm{kB}$ pathway, a master proinflammatory switch, is of great importance to the entire process from simple steatosis to steatohepatitis (14). The binding of various homo- or heterodimers, such as p 65 formed by NF- $\mathrm{KB}$ proteins to a consensus sequence of NF- $\mathrm{KB}$, usually regulates the transcription of many factors functioning in NAFLD (15). Furthermore, the NF- $\kappa$ B pathway is reported to be activated by a cascade of downstream signals due to the activation of TLR4, which further activates the production of tumor necrosis factor (TNF)- $\alpha$, interleukin (IL)- 6 and other proinflammatory molecules (16). Studies have shown that TNF- $\alpha$ acts as a key role in NAFLD progression both in humans and animals (17), and anti-TNF- $\alpha$ drugs improve HFD-induced alterations of the liver (18). For IL-6, due to its ability to reduce oxidative stress and avoid mitochondrial dysfunction, it was initially thought to be a hepatoprotective factor in hepatic steatosis $(19,20)$, which was confirmed by further studies in other liver disease models (21-23). Later, it was reported that IL-6 increased in human nonalcoholic steatohepatitis, and deficiency of IL-6 inhibited the progression of NAFLD $(24,25)$.

Metformin, first introduced in the 1950s in the clinic, is an effective drug for NAFLD treatment, although its efficacy is limited and it has considerable side effects (26). In addition, berberine (BBR) isolated from Rhizoma Coptidis has been reported to have anti-diabetic and anti-hyperlipidemic effects (27-29). Furthermore, numerous studies have demonstrated that BBR can attenuate hepatic steatosis and hyperlipidemia by inhibiting the synthesis and accumulation of lipid in hepatocytes, which prevents the development of NAFLD $(30,31)$. Therefore, both drugs have effects on the treatment of NAFLD (32-34). However, the specific mechanism remains unclear.

C-C motif ligand 19 (CCL19), a CC-type chemokine also known as macrophage inflammatory protein- $3 \beta$ (MIP-3 $\beta$ ), is produced by macrophages, dendritic cells and other different cells, and it usually binds to CC chemokine receptor 7 (CCR7). CCL19 has been implicated in chronic inflammation, lymphoid neogenesis, and dendritic extension $(35,36)$. Studies have indicated that lack of CCL19-CCR7 signaling has a protective effect on diet-induced obesity and insulin resistance (37). However, further research on the effect of CCL19 on fatty liver disease is still lacking.

In this paper, high expression levels of CCL19, TLR4, NF- $\kappa$ B-p56, IL-6, and TNF- $\alpha$ were detected in NAFLD patients and increased with the severity of NAFLD. This suggested that CCL19 may be implicated in NAFLD progression. In a rat model of NAFLD, dyslipidemia and the liver histopathology of steatosis were improved by metformin and BBR. Furthermore, gene expression analysis uncovered that high expression of CCL19 and related factors were also significantly decreased by metformin and BBR, whereas p-AMPK levels were increased. Therefore, we speculate that metformin and BBR improves NAFLD may through AMPK activation, and the inhibition of CCL19 is related to NAFLD treatment.

\section{Materials and methods}

Samples of peripheral blood and liver tissues of patients with fatty liver diseases. Based on specific criteria, the severity of NAFLD was classified as absent, mild NAFLD (Liver echogen slightly increased, good visualization of intrahepatic vessels and diaphragms), moderate NAFLD (Increased liver echogenicity, disappearance of intrahepatic vascular echo lines) and severe NAFLD (Significant increase in liver echogenicity, poor penetration of the right hepatic lobe, and blurred vision of the diaphragm) $(38,39)$. After obtaining written and informed consent, peripheral blood and liver tissues were collected from 30 healthy volunteers, 30 mild NAFLD patients and 30 severe NAFLD patients. All of the patients enrolled in this study were treated in Putuo District People's Hospital of Shanghai City (Shanghai, China). After processing the samples, ELISA and western blot analyses were carried out to examine relative gene or factor expression. All experiments in this study were approved by the Ethics Committee of Putuo District People's Hospital of Shanghai City.

Experimental group. For follow-up animal experiments, a rat model of fatty liver was induced by feeding a high-fat diet (HFD). Sixty Sprague-Dawley (SD) rats from the Experimental Animal Center (Shanghai, China) were housed in a room with a constant temperature of $22-25^{\circ} \mathrm{C}$, a $12 / 12 \mathrm{~h}$ light/dark cycle and a relative humidity of $60 \pm 10 \%$. The sixty rats were fed randomly with normal chow diet $(n=12)$ or HFD $(n=48)$ for 4 weeks to induce NAFLD. Then, the 48 HFD-fed rats were further randomized and continually fed with HFD (HFD group), with HFD and treatment of $150 \mathrm{mg} / \mathrm{kg} / \mathrm{d}$ metformin (HFD + metformin), with HFD and treatment of $250 \mathrm{mg} / \mathrm{kg} / \mathrm{d}$ BBR (HFD + BBR), or with HFD and treatment of $150 \mathrm{mg} / \mathrm{kg} / \mathrm{d}$ metformin plus $250 \mathrm{mg} / \mathrm{kg} / \mathrm{d}$ BBR (HFD + metformin + BBR) for 4 and 8 weeks. Additionally, rats fed a normal chow diet constantly without exposure to HFD were regarded as controls. At the end of 4 and 8 weeks of treatment, the serum of these rats was collected for biochemical and specific ELISA testing, while the liver tissues obtained at 8 weeks were utilized for hematoxylin and eosin (H\&E) staining, RT-PCR as well as western blot analyses. All of the experimental procedures were approved by the Experimental Animal Research Committee of Putuo District People's Hospital of Shanghai (Shanghai, China).

Reverse transcription-quantitative polymerase chain reaction (RT-qPCR) assay. TRIzol reagent (Invitrogen; Thermo Fisher Scientific, Inc., Waltham, MA, USA) was applied to isolate the total RNA from the liver tissues. After quantification, the integrity of RNA was confirmed by electrophoresis using a $1 \%$ agarose gel. Then, using a reverse transcriptase kit (Fermentas; Thermo Fisher Scientific, Inc., Pittsburgh, PA, USA), cDNA was generated from isolated RNA through reverse transcription. Subsequently, using a SYBR Green PCR kit (Thermo Fisher Scientific, Inc.) and cDNA as the template, a $25 \mu 1$ volume of RT-PCR reaction was assessed by a ABI Prism 7300 real-time PCR system (Applied Biosystems; Thermo Fisher Scientific, Inc., Waltham, MA, USA). After the procedure, using GAPDH as an internal control and using the $2^{-\Delta \Delta C q}$ method (40), the mRNA expression of CCL19, SREBP-1c, FAS, TLR4 and NF- $\mathrm{kB}-\mathrm{p} 65$ was determined. The sequences of primers used were as follows: GAPDH forward (F): 5'-GGAGTCTACTGGCGTCTTCAC-3' and reverse (R): 5'-ATGAGCCCTTCCACGATGC-3'; CCL19 F: 5'-CCT TCCGCTACCTTCTTATC-3' and R: 5'-CTCTTCTGGTCC TTGGTTTC-3'; SREBP-1c F: 5'CGCTACCGTTCCTCTATC 
AATG3' and R: 5'-TCTGGTTGCTGTGCTGTAAG-3'; FAS F: 5'-TGGTTCATTCCGTGACTG-3' and R: 5'-TGTCCT TCGGTTCTTTCC-3'; TLR4 F: 5'-ATGCTAAGGTTGGCA CTCTC-3' and R: 5'-CAGGCAGGAAAGGAACAATG-3'; NF-кB-p65 F: 5'-AGACCTGGAGCAAGCCATTAG-3' and R: 5'-CGGACCGCATTCAAGTCATAG-3'. The RT-PCR procedure was as follows: $95^{\circ} \mathrm{C}$ for $10 \mathrm{~min}$; followed by $95^{\circ} \mathrm{C}$ for $15 \mathrm{sec}, 60^{\circ} \mathrm{C}$ for $45 \mathrm{sec}$ for 40 cycles; $95^{\circ} \mathrm{C}$ for $15 \mathrm{sec}$ and $60^{\circ} \mathrm{C}$ for $1 \mathrm{~min}$ for one cycle; and $95^{\circ} \mathrm{C}$ for $15 \mathrm{sec}$ and $60^{\circ} \mathrm{C}$ for $15 \mathrm{sec}$ for one cycle (41).

Western blot analysis. The collected liver tissues were cut into tiny pieces, lysed by RIPA buffer (Beijing Solarbio Science $\&$ Technology Co., Ltd., Beijing, China) containing protease and phosphatase inhibitors, and completely homogenized with a homogenizer. Using a prechilled centrifuge, the proteins in the supernatant of the solution were acquired after centrifugation for $15 \mathrm{~min}$ at $12,000 \mathrm{x} \mathrm{g}$. Later, following quantification with a BCA kit (Thermo Fisher Scientific, Inc.), the proteins were separated by electrophoresis using 10 and $15 \%$ gels, followed by a semi-dry transfer onto polyvinylidene fluoride (PVDF) membranes (EMD Millipore, Billerica, MA, USA). Next, the membranes were blocked with $5 \%$ skim milk (BD Biosciences, Franklin Lakes, NJ, USA) for $1 \mathrm{~h}$ at room temperature (or overnight at $4^{\circ} \mathrm{C}$ ). After blocking, several specific primary antibodies against the target genes CCL19 (1:500, cat. no. bs-2454R; BIOSS, Beijing, China), TLR4 (1:500, cat. no. Sc-293072; Santa Cruz Biotechnology, Inc., Dallas, TX, USA), NF-кB-p65 (1:1,000, cat. no. 8242; Cell Signaling Technology, Inc., Danvers, MA, USA), SREBP-1c (1:1,000, cat. no. Ab28481; Abcam, Cambridge, MA, USA), FAS (1:1,000, cat. no. Ab82419; Abcam), AMPK (1:800, Ab110036; Abcam), p-AMPK (1:1,000, cat. no. AF3423; Affinity Biosciences, Zhenjiang, China), and GAPDH (1:2,000, cat. no. 5174; Cell Signaling Technology, Inc.) were incubated with the membranes at $4^{\circ} \mathrm{C}$ overnight with gentle shaking (or $2 \mathrm{~h}$ at room temperature). Subsequently, after 5-6 washes, the membranes were incubated with corresponding secondary antibodies (1:1,000 dilution; Beyotime, Shanghai, China) according to the sources of primary antibodies for $1 \mathrm{~h}$ at $37^{\circ} \mathrm{C}$. Finally, after 5 min of incubation of the chemiluminescence detection reagent (cat. no. WBKLS0100; EMD Millipore) in the dark, the bands of target proteins were visualized through an ECL imaging system (Tanon, Shanghai, China).

Enzyme-linked immunosorbent assay (ELISA). The peripheral blood samples from patients and treated rats were harvested. Following centrifugation at 2,000-3,000 $\mathrm{x}$ g for approximately $20 \mathrm{~min}$, the supernatants of the samples were obtained. Using a double antibody sandwich ELISA kit, the concentrations of CCL19, IL-6 and TNF- $\alpha$ in the supernatant of peripheral blood were detected.

$H \& E$ staining. As previously described, the liver tissues collected from treated rats were embedded in paraffin and sliced into 4-7 $\mu \mathrm{m}$ sections by a paraffin slicer. The liver sections were then stained with double dyes of hematoxylin and eosin (BASO) (42). After staining, each section was assessed and photographed by a 10x200 light microscope. Following the criteria, the severity of fatty liver was scored: Grade 0 , no steatosis; grade 1 , less than $10 \%$ of hepatic parenchyma is fatty hepatocytes; grade 2, occupying 10-30\%; grade 3, occupying $30-60 \%$; grade 4 , more than $60 \%$ is fatty hepatocytes (43).

Biochemical analysis. After 4 and 8 weeks of drug treatment, the serum of these rats was collected for biochemical analysis. A specific AST kit (C010-1) was used to analyze the activity of AST in serum following the recommended protocol. Likewise, the activity of ALT, TG and TC was also respectively detected by specific ALT (C009-1), TG (A110-2) and TC (A111-2) testing kit which were provided by Nanjing Jiancheng Bioengineering Institute (Nanjing, China).

Statistical analysis. GraphPad Prism 7.0 software (GraphPad Software, Inc., La Jolla, CA, USA) was used to carry out statistical analyses. One way analysis of variance followed by Tukey's post hoc test was used to examine multiple comparisons. With at least three independent experiments, all results were presented as the mean \pm standard deviation, and $\mathrm{P}<0.05$ was considered to indicate a statistically significant difference.

\section{Results}

High levels of CCL19, TLR4, NF- $\kappa B-p 65, I L-6$, and TNF- $\alpha$ in NAFLD patients. Studies have correlated signal pathways (TLR4/NF- $\kappa B-p 65)$ and proinflammatory cytokines (IL-6/TNF- $\alpha$ ) to NAFLD progression $(13,14,17,24)$. Thus, peripheral blood samples were collected from 30 healthy controls, 30 mild NAFLD patients, and 30 severe NAFLD patients. After preprocessing, the concentrations of CCL19, IL-6 and TNF- $\alpha$ in these samples were measured by specific ELISA. As shown in Fig. 1A-C, the levels of CCL19, IL-6 and TNF- $\alpha$ were much higher in the blood of NAFLD patients than in that of healthy controls. Furthermore, the levels of CCL19, IL-6 and TNF- $\alpha$ progressively increased with the severity of fatty liver. Through western blot analysis, we found that the protein levels of CCL19, TLR4 and NF-kB-p56 in liver tissues collected from NAFLD patients were also markedly increased with the severity of fatty liver (Fig. 1D). These data indicate that CCL19, TLR4, NF-kB-p65, IL-6, and TNF- $\alpha$ may function in fatty liver.

The levels of plasma AST, ALT, TC and TG in HFD rats and drug-treated HFD rats. To verify the rat model of NAFLD and the effect of metformin or BBR on NAFLD, the levels of AST, ALT, TC and TG in the sera of HFD and drug-treated HFD rats were measured at 4 and 8 weeks by specific biochemical testing. As shown in Fig. 2, HFD rats displayed dyslipidemia with elevated levels of plasma AST, ALT, TC and TG, which was similar to the human dyslipidemia of NAFLD. In addition, metformin or BBR led to an inhibiting effect on HFD-induced dyslipidemia, and the joint effect of the two drugs was better. These results suggest that dyslipidemia of NAFLD patients can be simulated by HFD feeding and improved by metformin and BBR treatment.

Liver histopathology of HFD rats and drug-treated HFD rats. To further study HFD-induced NAFLD as well as the effects of the two drugs on NAFLD, the liver histopathology of the treated rats was analyzed by $\mathrm{H} \& \mathrm{E}$ staining at 8 weeks. As shown in Fig. 3, we found significantly higher microvesicular 
A

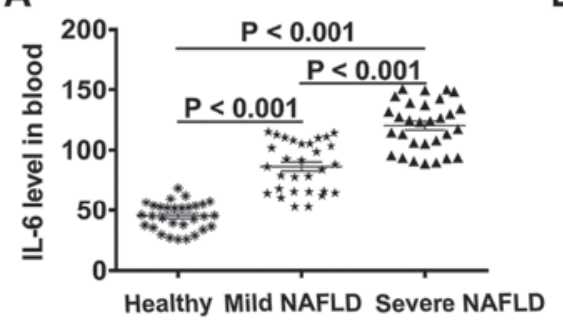

B

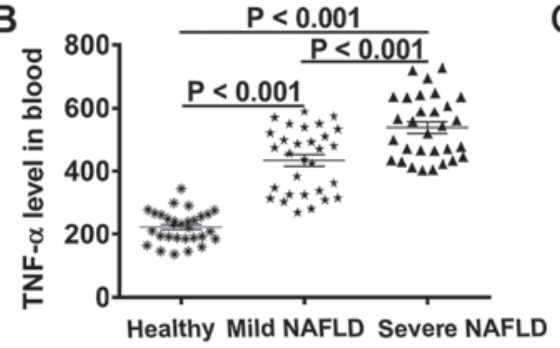

C

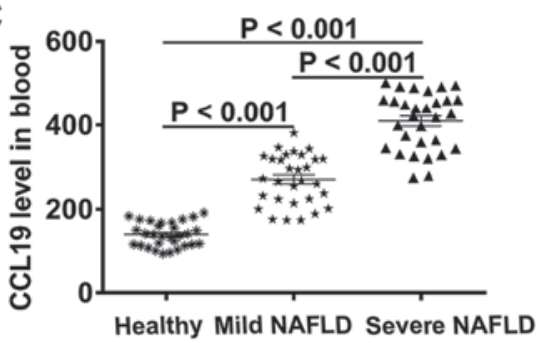

D
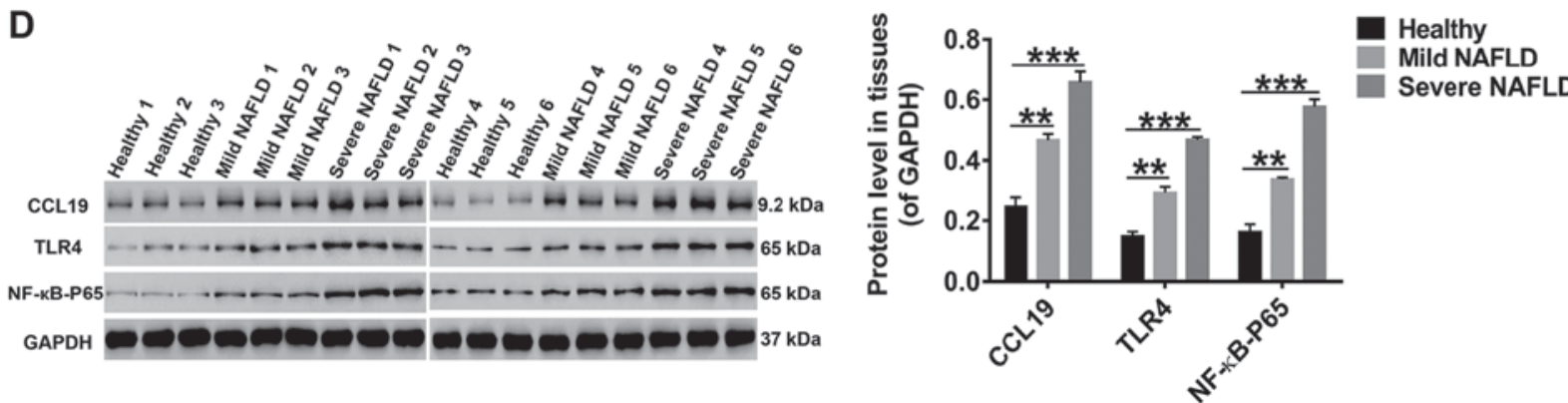

Figure 1. High levels of CCL19, TLR4, NF-kB-p65, IL-6 and TNF- $\alpha$ in NAFLD patients. Following processing, the concentrations of (A) IL-6, (B) TNF- $\alpha$ and (C) CCL19 in the blood were detected by ELISA. (D) The protein levels of CCL19, TLR4 and NF-kB-p65 in liver tissues were quantified by western blot analysis. All data are presented as the mean \pm standard deviation. ${ }^{* *} \mathrm{P}<0.01$ and ${ }^{* * *} \mathrm{P}<0.001$ as indicated. CCL19, C-C motif ligand 19; TLR4, Toll-like

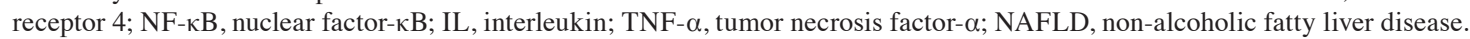
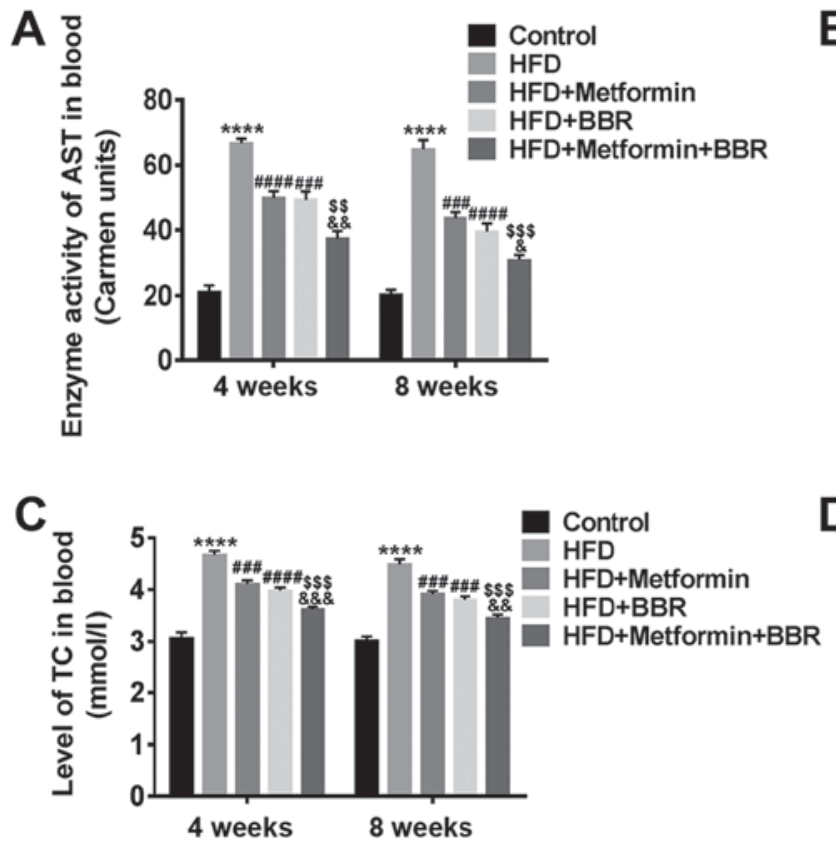

Figure 2. Levels of plasma AST, ALT, TC and TG in HFD rats and drug-treated HFD rats. The serum of Control, HFD, HFD + metformin, HFD + BBR and HFD + metformin + BBR rats was obtained at 4 and 8 weeks. Then, the levels of plasma (A) AST, (B) ALT, (C) TC and (D) TG in these rats were analyzed by specific biochemical testing. The data are presented as the mean \pm standard deviation. ${ }^{* * * *} \mathrm{P}<0.0001$ vs. Control; ${ }^{\# \# \#} \mathrm{P}<0.001$ and ${ }^{\# \# \# \#} \mathrm{P}<0.0001$ vs. HFD; ${ }^{\$ \$} \mathrm{P}<0.01$, ${ }^{\$ \$ \$} \mathrm{P}<0.001$ and ${ }^{\$ \$ \$ \$} \mathrm{P}<0.0001$ vs. HFD + metformin; ${ }^{\circledR} \mathrm{P}<0.05,{ }^{\& \&} \mathrm{P}<0.01$ and ${ }^{\& \& \&} \mathrm{P}<0.001$ vs. HFD + BBR. AST, aspartate aminotransferase; $\mathrm{ALT}$, alanine aminotransferase; TC, total cholesterol; TG, triglyceride; HFD, high-fat diet; BBR, berberine.

and macrovesicular steatosis in liver tissues after HFD feeding than in the control, which further evidenced that HFD feeding can simulate steatosis to human NAFLD (Fig. 3A and B). In Fig. 3C-E, HFD-induced steatosis was remarkably eliminated by metformin or BBR treatment, and the joint effect of the two drugs was better. This further demonstrates that metformin and BBR contribute to NAFLD treatment.
The concentrations of CCL19, IL- 6 and TNF- $\alpha$ in serum of $H F D$ rats and drug-treated HFD rats. Experimental animals fed with HFD are considered good models of NAFLD. Here, we further explored the effect of CCL19 in these models. After 4 and 8 weeks of treatment, the concentrations of CCL19, IL-6 and TNF- $\alpha$ in the sera of these treated rats were measured by specific ELISA. As shown in Fig. 4, elevated levels of CCL19, 
A

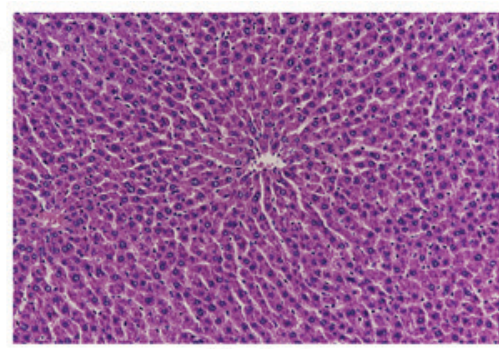

Control

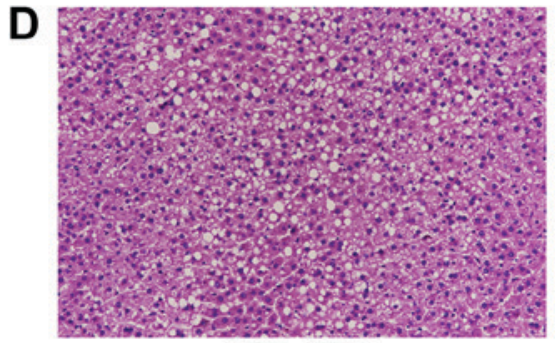

HFD+BBR

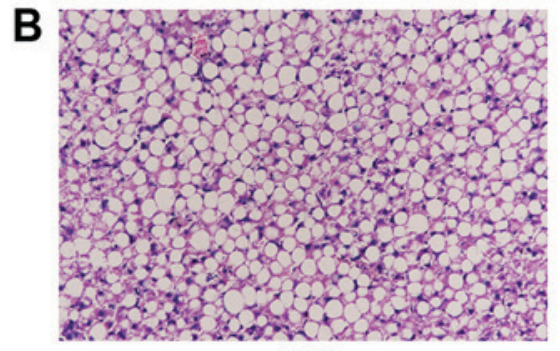

HFD

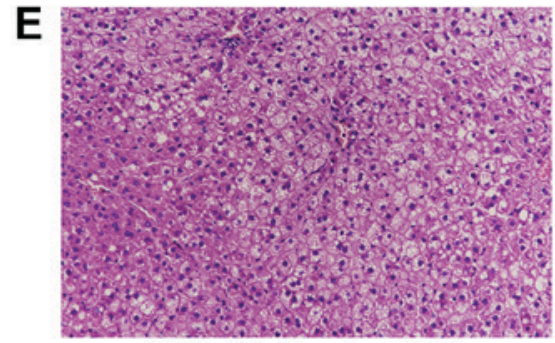

HFD+Metformin+BBR

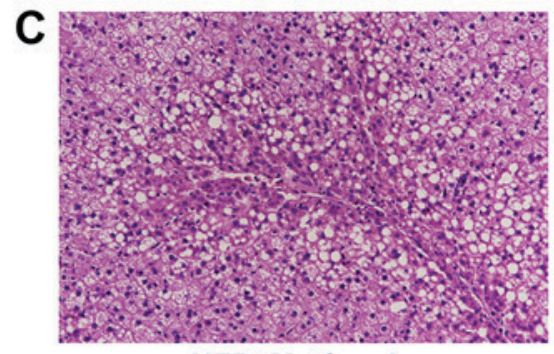

HFD+Metformin

Figure 3. Liver histopathology of HFD- and drug-treated rats. Following 8 weeks, liver tissues of Control, HFD, HFD + metformin, HFD + BBR and HFD + metformin + BBR rats were stained with hematoxylin and eosin and then imaged using a microscope at magnification, x200. (A) Normal liver tissues of Control rats. (B) Microvesicular and macrovesicular steatosis were evident, with scattered necroinflammatory foci in HFD rats. (C) Steatosis was inhibited by metformin in the HFD + metformin group, and (D) by BBR in the HFD + BBR group. (E) The inhibition of steatosis was more marked when metformin treatment was combined with BBR. HFD, high-fat diet; BBR, berberine.
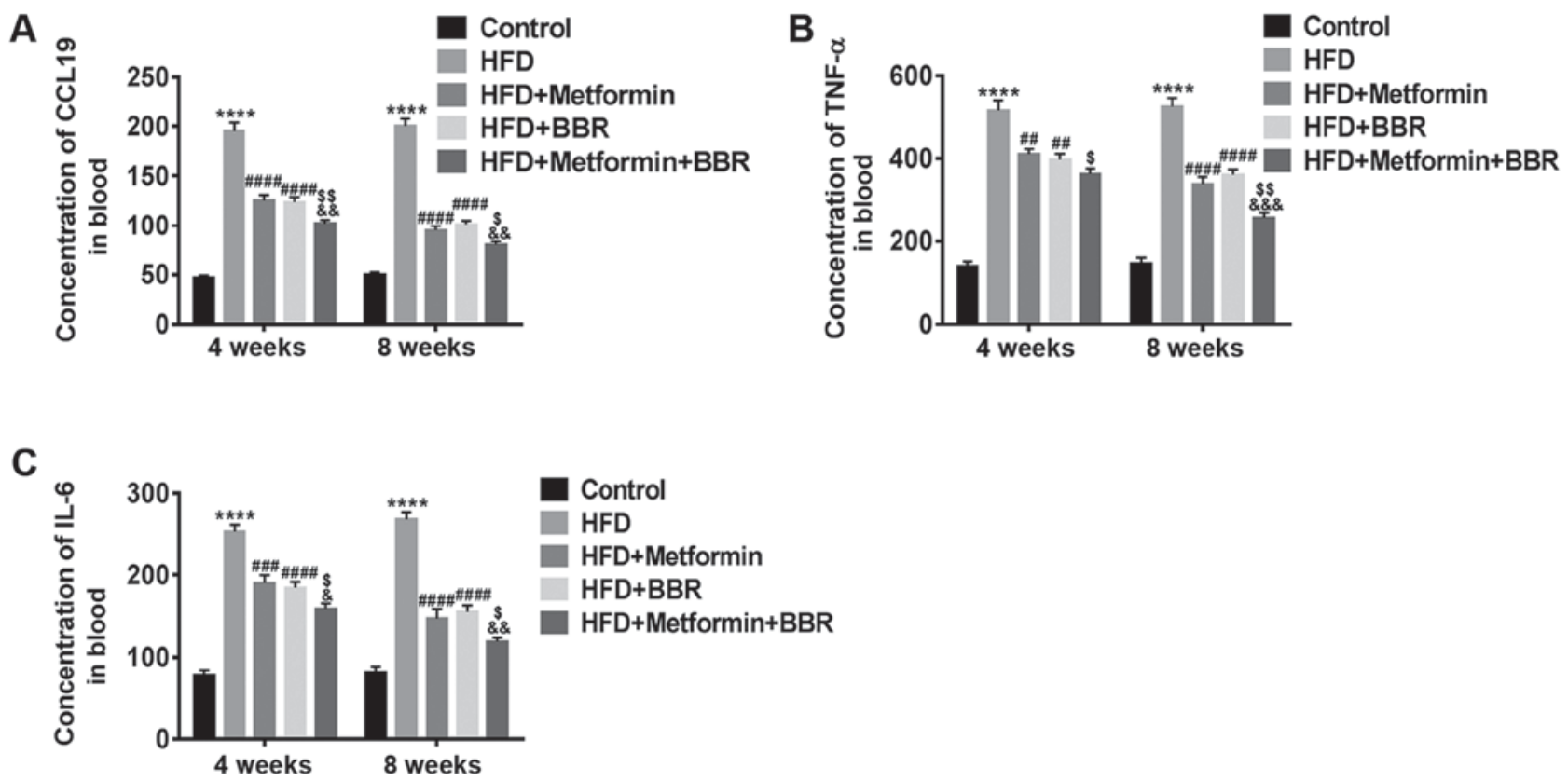

Figure 4. Concentrations of CCL19, IL-6 and TNF- $\alpha$ in the sera of HFD- and drug-treated HFD rats. The serum concentrations of (A) CCL19, (B) TNF- $\alpha$ and (C) IL-6 in Control, HFD, HFD + metformin, HFD + BBR and HFD + metformin + BBR rats were measured by ELISA at 4 and 8 weeks. The data are expressed as the mean \pm standard deviation. ${ }^{* * * *} \mathrm{P}<0.0001$ vs. Control; ${ }^{\# \#} \mathrm{P}<0.01,{ }^{\# \# \#} \mathrm{P}<0.001$ and ${ }^{\# \# \# *} \mathrm{P}<0.0001$ vs. HFD; ${ }^{\$} \mathrm{P}<0.05$ and ${ }^{\$ S} \mathrm{P}<0.01$ vs. HFD + metformin; ${ }^{\&} \mathrm{P}<0.05$, ${ }^{\&} \mathrm{P}<0.01$ and ${ }^{\& \& \&} \mathrm{P}<0.001$ vs. HFD + BBR. CCL19, C-C motif ligand 19; IL, interleukin; TNF- $\alpha$, tumor necrosis factor- $\alpha$; HFD, high-fat diet; BBR, berberine.

IL-6 and TNF- $\alpha$, similar to those observed in NAFLD patients, were also present in HFD rats. Metformin and BBR markedly inhibited the HFD-induced levels of CCL19, IL- 6 and TNF- $\alpha$. These results demonstrate that the release of CCL19 and the proinflammatory cytokines IL- 6 and TNF- $\alpha$ may enhance the progression of NAFLD and is inhibited by metformin and BBR, which may be closely related to the treatment of NAFLD.
The expression of CCL19, TLR4, NF- $\kappa B-p 65$, SREBP-1c, $F A S, p-A M P K$, and AMPK in the liver tissues of HFD rats and drug-treated HFD rats. For further study, the levels of CCL19, signaling pathways (TLR4/NF-кB-p65) and lipid metabolism factors (SREBP-1c and FAS) in the liver tissues of these treated rats were quantified by RT-PCR and western blot. As shown in Fig. 5, the HFD-induced expression 


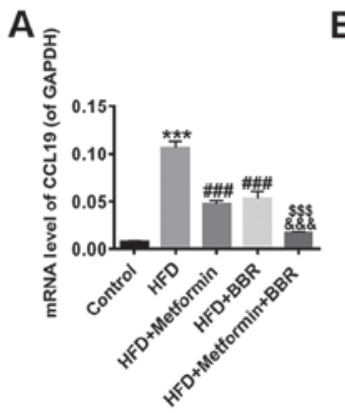

$\mathbf{F}$

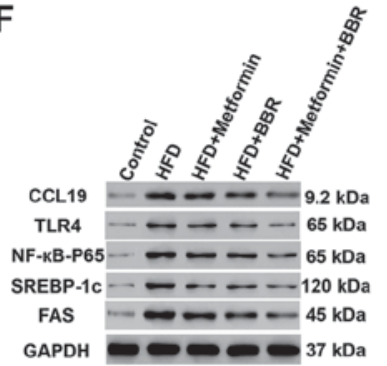

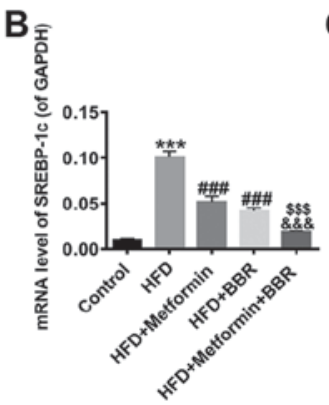

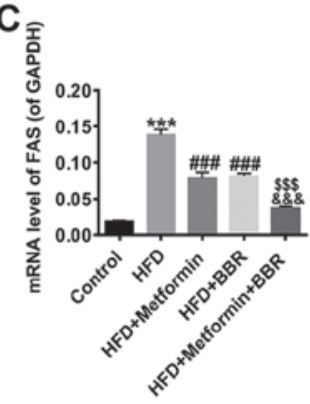

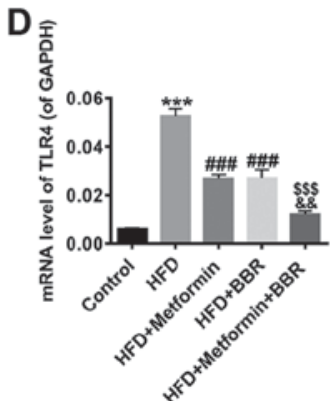

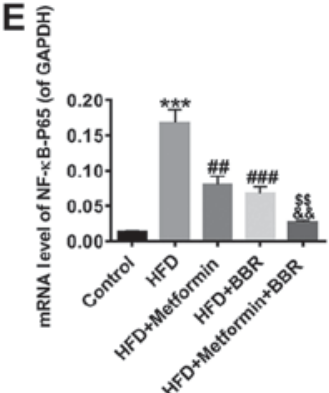

G
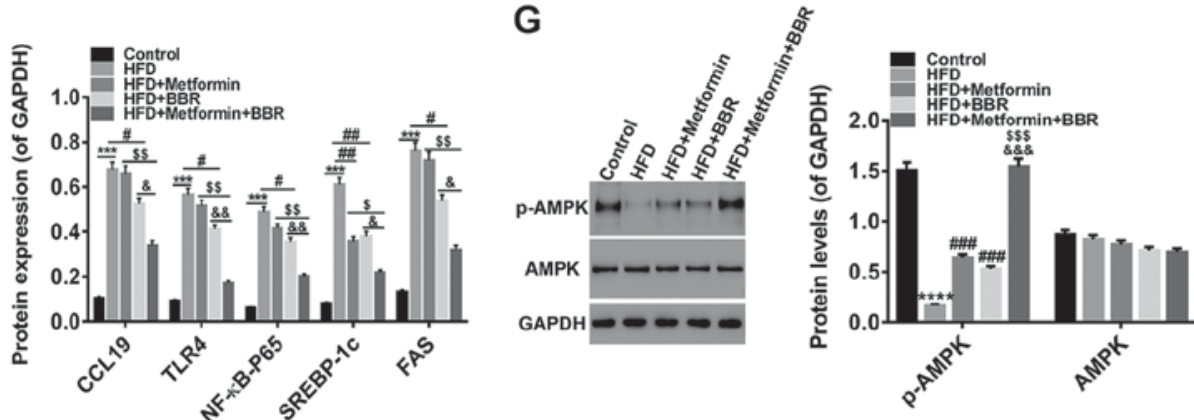

Figure 5. Expression of CCL19, TLR4, NF-кB-p65, SREBP-1c, FAS, p-AMPK and AMPK in the liver tissues of HFD- and drug-treated HFD rats Following 8 weeks of drug treatment, the mRNA levels of (A) CCL19, (B) SREBP-1c, (C) FAS, (D) TLR4 and (E) NF-kB-p65 were quantified by reverse transcription-quantitative polymerase chain reaction, while (F) western blotting was applied to detect the protein levels. (G) In addition, the levels of p-AMPK and AMPK proteins were detected. All data are presented as the mean \pm standard deviation. ${ }^{* * * *} \mathrm{P}<0.001$ and ${ }^{* * * *} \mathrm{P}<0.0001$ vs. Control; ${ }^{\#} \mathrm{P}<0.05$, ${ }^{\# \#} \mathrm{P}<0.01$ and

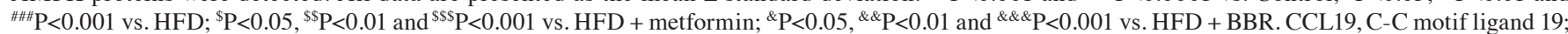

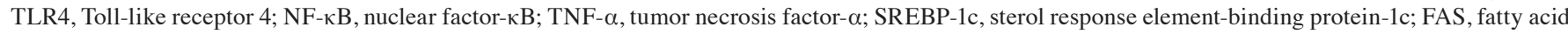
synthase; p-, phosphorylated; AMPK, adenosine monophosphate-activated protein kinase; HFD, high-fat diet; BBR, berberine.

of CCL19, TLR4, NF-кB-p65, SREBP-1c, and FAS was inhibited by the two drugs at both the transcriptional and translational levels, and the joint effect of the two drugs was better. In addition, HFD suppression of phosphorylated-AMPK (p-AMPK) levels was significantly increased by treatment with the two drugs, whereas AMPK expression was unchanged. These results further clarified that the expression of CCL19, TLR4, NF-kB-p65, SREBP-1c, FAS, and $\mathrm{p}$-AMPK was related to the NAFLD process. Metformin and BBR improved NAFLD may through the activation of AMPK and the inhibition of TLR4/NF- $\mathrm{BB}-\mathrm{p} 65$ signaling. Meantime, CCL19 expression was inhibited by metformin and BBR, indicating that inhibition of CCL19 may be benefit NAFLD treatment.

\section{Discussion}

Fatty liver disease remains the most common chronic liver disease and is a serious threat to human health. Previous research has demonstrated that various factors, including cytokines and chemokines, are involved in fatty liver diseases. For instance, high expression of CCL2 (also known as MCP-1) was noted in the steatotic livers of NAFLD patients (44) and could mediate the inflammatory response of NAFLD (45). In addition, CCL5 (also known as RANTES) was also expressed highly in NAFLD patients (46), and it has been reported that CCL20 (also known as MIP-3 $\alpha$ ) was enriched in the necrotic regions of liver portions from hepatitis patients (47).

In our study, the levels of CCL19, TLR4/NF-kB-p65 and IL-6/TNF- $\alpha$ were markedly increased in NAFLD patients,

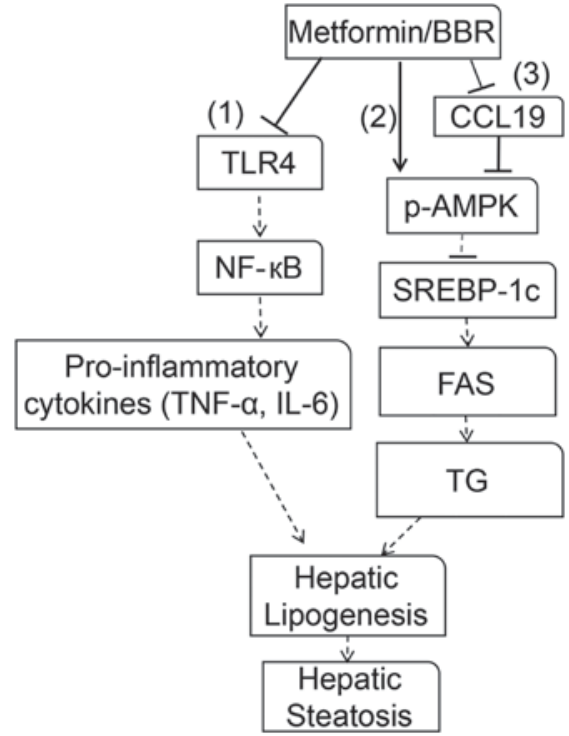

Figure 6. Molecular mechanisms of metformin or BBR in reverting dysfunction in NAFLD. Metformin or BBR induced a series of alterations, including proinflammatory cytokine production and lipogenesis in the liver. In protecting NAFLD, metformin or BBR is directed toward several pathways. (1) Metformin or BBR inhibits TLR4, which further inhibits NF-кB-p65 pathway activation, thereby reducing IL-6/TNF- $\alpha$ production. (2) Metformin or BBR phosphorylates AMPK, and the activation of AMPK inhibits SREBP-1c expression, which further inhibits lipogenesis by inhibiting FAS expression. (3) Metformin or BBR phosphorylates AMPK by regulating CCL19. Hepatic lipogenesis and hepatic steatosis are suppressed via these pathways. BBR, berberine; NAFLD, non-alcoholic fatty liver disease; TLR4,

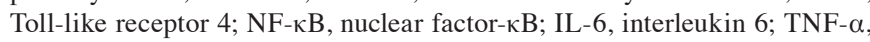
tumor necrosis factor- $\alpha$; p-, phosphorylated; AMPK, adenosine monophosphate-activated protein kinase; SREBP-1c, sterol response element-binding protein-1c; FAS, fatty acid synthase; TG, triglyceride; CCL19, C-C motif ligand 19 
indicating that these molecules may be implicated in NAFLD progression. Furthermore, high release of CCL19 may offer a novel diagnostic blood indicator of NAFLD. In a rat model of NAFLD, metformin or BBR could ameliorate NAFLD by reducing the dyslipidemia and steatosis, and the effect of the two drugs combined was much better. Additionally, high levels of CCL19, TLR4/NF-кB-p65, SREBP-1c, FAS and IL-6/TNF- $\alpha$ were also remarkably decreased, whereas HFD-suppressed p-AMPK levels were increased by drug treatment. Based on these data, we inferred that metformin and BBR treatment improved NAFLD may through the activation of AMPK and the inactivation of TLR4/NF- $\mathrm{B}-\mathrm{p} 65$ signaling. Meantime, metformin and BBR could inhibit CCL19, which related CCL19 inhibition to the treatment of NAFLD. As shown in Fig. 6, the experiments indicated

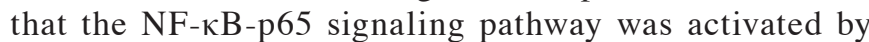
upstream TLR4 via signal transduction, which further promoted the secretion of proinflammatory cytokines IL-6 and TNF- $\alpha$ (48). By regulating the chronic inflammatory response, IL- 6 and TNF- $\alpha$ have been demonstrated to be essential for fatty liver $(49,50)$. In addition, SREBP-1c was reported to upregulate the expression of several prolipogenic genes, such as FAS, and be responsible for the modulation of TG synthesis in the liver. AMPK has been suggested to regulate cellular metabolism, such as lipid metabolism (51); by downregulating SREBP-1c, AMPK activation inhibits FAS expression, enhancing TG deposition and further causing hepatic lipogenesis and hepatic steatosis (52). Thus, TLR4/NF- $\mathrm{B}-\mathrm{p} 65 / \mathrm{IL}-6 / \mathrm{TNF}-\alpha$ and p-AMPK/SREBP-1c/FAS play important roles in NAFLD progression, which is consistent with the results of our study. Furthermore, the CCL19 promoter has been reported to have two putative NF- $\mathrm{NB}$ binding sites, and CCL19 expression is regulated by several transcription factors, including $\mathrm{NF}-\kappa \mathrm{B}$. Activity of the CCL19 promoter was induced by ectopic expression of TLR3/4 (15). Therefore, combining our results that CCL19 expression was inhibited by metformin and BBR, it is likely that CCL19 functions in NAFLD, which probably involve TLR4/NF-кB-p65 pathway, but further studies are needed. Thus, targeting CCL19 is potential to provide a novel effective therapy for NAFLD.

In conclusion, we found that metformin and BBR could improve NAFLD by p-AMPK activation and TLR4/NF- $\kappa$ B-p65 inactivation. Meantime, CCL19 levels were inhibited by metformin and BBR, indicating that CCL19 inhibition may contribute to NAFLD treatment. Although the mechanisms are still unclear, these data reveal that CCL19 may act as a new target for therapy of fatty liver diseases.

\section{Acknowledgements}

Not applicable.

\section{Funding}

The present study was funded by Shanghai Grassroots Senior Experts in Traditional Chinese Medicine Heritage Research Studio Construction Projects (grant no. JCZYGZS-020) and Putuo District People's Hospital Fund for Distinguished Young Scholars (grant no. PRYYC2016-A001).

\section{Availability of data and materials}

All data generated or analyzed during this study are included in this published article.

\section{Authors' contributions}

JZ and JH conceived and designed the study. JZ, YW, XW, PT, YY, SG and DH performed the experiments. JZ and JH wrote the manuscript. All authors read and approved the final manuscript.

\section{Ethics approval and consent to participate}

All experiments in the present study were approved by the Ethics Committee of Putuo District People's Hospital of Shanghai City (Shanghai, China); written informed consent was obtained from all patients. All of the experimental procedures involving animals were approved by the Experimental Animal Research Committee of Putuo District People's Hospital of Shanghai.

\section{Patient consent for publication}

Not applicable.

\section{Competing interests}

The authors declare that they have no competing interests.

\section{References}

1. Angulo P: Nonalcoholic fatty liver disease. N Engl J Med 346: 1221-1231, 2002

2. Ding RB, Bao J and Deng CX: Emerging roles of SIRT1 in fatty liver diseases. Int J Biol Sci 13: 852-867, 2017.

3. Vernon G, Baranova A and Younossi ZM: Systematic review: The epidemiology and natural history of non-alcoholic fatty liver disease and non-alcoholic steatohepatitis in adults. Aliment Pharmacol Ther 34: 274-285, 2011.

4. Alisi A, Cianfarani S, Manco M, Agostoni C and Nobili V: Non-alcoholic fatty liver disease and metabolic syndrome in adolescents: Pathogenetic role of genetic background and intrauterine environment. Ann Med 44: 29-40, 2012.

5. Perticone M, Cimellaro A, Maio R, Caroleo B, Sciacqua A, Sesti G and Perticone F: Additive effect of non-alcoholic fatty liver disease on metabolic syndrome-related endothelial dysfunction in hypertensive patients. Int J Mol Sci 17: 456, 2016.

6. Cohen JC, Horton JD and Hobbs HH: Human fatty liver disease: Old questions and new insights. Science 332: 1519-1523, 2011.

7. Wu KT, Kuo PL, Su SB, Chen YY, Yeh ML, Huang CI, Yang JF, Lin CI, Hsieh MH, Hsieh MY, et al: Nonalcoholic fatty liver disease severity is associated with the ratios of total cholesterol and triglycerides to high-density lipoprotein cholesterol. J Clin Lipidol 10: 420-425, 2016.

8. Kotronen A, Peltonen M, Hakkarainen A, Sevastianova K, Bergholm R, Johansson LM, Lundbom N, Rissanen A, Ridderstråle M, Groop L, et al: Prediction of non-alcoholic fatty liver disease and liver fat using metabolic and genetic factors. Gastroenterology 137: 865-872, 2009.

9. Zhou G, Myers R, Li Y, Chen Y, Shen X, Fenyk-Melody J, Wu M, Ventre J, Doebber T, Fujii N, et al: Role of AMP-activated protein kinase in mechanism of metformin action. J Clin Invest 108: 1167-1174, 2001.

10. Walker AK and Näär AM: SREBPs: Regulators of cholesterol/lipids as therapeutic targets in metabolic disorders, cancers and viral diseases. Clin Lipidol 7: 27-36, 2012.

11. Horton JD: Sterol regulatory element-binding proteins: Transcriptional activators of lipid synthesis. Biochem Soc Trans 30: 1091-1095, 2002. 
12. Bricambert J, Miranda J, Benhamed F, Girard J, Postic C and Dentin R: Salt-inducible kinase 2 links transcriptional coactivator p300 phosphorylation to the prevention of ChREBP-dependent hepatic steatosis in mice. J Clin Invest 120: 4316-4331, 2010.

13. Kiziltas S, Ata P, Colak Y, Mesçi B, Senates E, Enc F, Ulasoglu C, Tuncer I and Oguz A: TLR4 gene polymorphism in patients with nonalcoholic fatty liver disease in comparison to healthy controls. Metab Syndr Relat Disord 12: 165-170, 2014

14. Zhao CY, Yan L, Wang YD, Wang W, Zhou JY and Zhen Z: Role of resistin in inflammation of hepatocytes in nonalcoholic steatohepatitis. Zhonghua Gan Zang Bing Za Zhi 17: 683-687, 2009 (In Chinese)

15. Pietilä TE, Veckman V, Lehtonen A, Lin R, Hiscott J and Julkunen I: Multiple NF-kappaB and IFN regulatory factor family transcription factors regulate CCL19 gene expression in human monocyte-derived dendritic cells. J Immunol 178: 253-261, 2007.

16. Zuany-Amorim C, Hastewell J and Walker C: Toll-like receptors as potential therapeutic targets for multiple diseases. Nat Rey Drug Discov 1: 797-807, 2002.

17. Hotamisligil GS, Shargill NS and Spiegelman BM: Adipose expression of tumor necrosis factor-alpha: Direct role in obesity-linked insulin resistance. Science 259: 87-91, 1993.

18. Pinto Lde F, Compri CM, Fornari JV, Bartchewsky W, Cintra DE, Trevisan M, Carvalho Pde O, Ribeiro ML, Velloso LA, Saad MJ, et al: The immunosuppressant drug, thalidomide, improves hepatic alterations induced by a high-fat diet in mice. Liver Int 30: 603-610, 2010.

19. El-Assal O, Feng H, Kim WH, Radaeva S and Gao B: IL-6-deficient mice are susceptible to ethanol-induced hepatic steatosis: IL-6 protects against ethanol-induced oxidative stress and mitochondrial permeability transition in the liver. Cell Mol Immunol 1: 205-211, 2004.

20. Teoh N, Field J and Farrell G: Interleukin-6 is a key mediator of the hepatoprotective and pro-proliferative effects of ischaemic preconditioning in mice. J Hepatol 45: 20-27, 2006.

21. Peters M, Blinn G, Jostock T, Schirmacher P, Meyer zum Büschenfelde KH, Galle PR and Rose-John S: Combined interleukin 6 and soluble interleukin 6 receptor accelerates murine liver regeneration. Gastroenterology 119: 1663-1671, 2000.

22. Blindenbacher A, Wang X, Langer I, Savino R, Terracciano L and Heim MH: Interleukin 6 is important for survival after partial hepatectomy in mice. Hepatology 38: 674-682, 2003.

23. Klein C, Wüstefeld T, Assmus U, Roskams T, Rose-John S, Müller M, Manns MP, Ernst $M$ and Trautwein C: The IL-6-gp130-STAT3 pathway in hepatocytes triggers liver protection in T cell-mediated liver injury. J Clin Invest 115: 860-869, 2005.

24. Mas E, Danjoux M, Garcia V, Carpentier S, Ségui B and Levade T: IL-6 deficiency attenuates murine diet-induced non-alcoholic steatohepatitis. PLoS One 4: e7929, 2009.

25. Wieckowska A, Papouchado BG, Li Z, Lopez R, Zein NN and Feldstein AE: Increased hepatic and circulating interleukin-6 levels in human nonalcoholic steatohepatitis. Am J Gastroenterol 103: 1372-1379, 2008

26. Lavine JE, Schwimmer JB, Van Natta ML, Molleston JP Murray KF, Rosenthal P, Abrams SH, Scheimann AO, Sanyal AJ, Chalasani N, et al: Effect of vitamin $\mathrm{E}$ or metformin for treatment of nonalcoholic fatty liver disease in children and adolescents: The TONIC randomized controlled trial. JAMA 305: 1659-1668, 2011.

27. Yin J, Xing $\mathrm{H}$ and Ye J: Efficacy of berberine in patients with type 2 diabetes mellitus. Cell Mol Immunol 57: 712-717, 2008.

28. Kong W, Wei J, Abidi P, Lin M, Inaba S, Li C, Wang Y, Wang Z, $\mathrm{Si}$ S, Pan H, et al: Berberine is a novel cholesterol-lowering drug working through a unique mechanism distinct from statins. Nat Med 10: 1344-1351, 2004.

29. Kong WJ, Zhang H, Song DQ, Xue R, Zhao W, Wei J, Wang YM, Shan N, Zhou ZX, Yang P, et al: Berberine reduces insulin resistance through protein kinase C-dependent up-regulation of insulin receptor expression. Metabolism 58: 109-119, 2009.

30. Brusq JM, Ancellin N, Grondin P, Guillard R, Martin S, Saintillan Y and Issandou M: Inhibition of lipid synthesis through activation of AMP kinase: An additional mechanism for the hypolipidemic effects of berberine. J Lipid Res 47: 1281-1288, 2006.

31. Chang X, Yan H, Fei J, Jiang M, Zhu H, Lu D and Gao X: Berberine reduces methylation of the MTTP promoter and alleviates fatty liver induced by a high-fat diet in rats. J Lipid Res 51: 2504-2515, 2010.
32. Yuan X, Wang J, Tang X, Li Y, Xia P and Gao X: Berberine ameliorates nonalcoholic fatty liver disease by a global modulation of hepatic mRNA and lncRNA expression profiles. J Transl Med 13: 24, 2015.

33. Yan HM, Xia MF, Wang Y, Chang XX, Yao XZ, Rao SX, Zeng MS, Tu YF, Feng R, Jia WP, et al: Efficacy of berberine in patients with non-alcoholic fatty liver disease. PLoS One 10: $\mathrm{e} 0134172,2015$.

34. Mazza A, Fruci B, Garinis GA, Giuliano S, Malaguarnera R and Belfiore A: The role of metformin in the management of NAFLD. Exp Diabetes Res 2012: 716404, 2012.

35. Luther SA, Bidgol A, Hargreaves DC, Schmidt A, Xu Y, Paniyadi J, Matloubian M and Cyster JG: Differing activities of homeostatic chemokines CCL19, CCL21 and CXCL12 in lymphocyte and dendritic cell recruitment and lymphoid neogenesis. Journal of Immunology 169: 424-433, 2002.

36. Yanagawa Y and Onoé K: CCL19 induces rapid dendritic extension of murine dendritic cells. Blood 100: 1948-1956, 2002

37. Sano T, Iwashita M, Nagayasu S, Yamashita A, Shinjo T, Hashikata A, Asano T, Kushiyama A, Ishimaru N, Takahama Y and Nishimura F: Protection from diet-induced obesity and insulin resistance in mice lacking CCL19-CCR7 signaling. Obesity (Silver Spring) 23: 1460-1471, 2015.

38. Borai IH, Shaker Y, Kamal MM, Ezzat WM, Ashour E, Afify M, Gouda W and Elbrashy MM: Evaluation of biomarkers in egyptian patients with different grades of nonalcoholic fatty liver disease. J Clin Transl Hepatol 5: 109-118, 2017.

39. Lee SH, Yun SJ, Kim DH, Jo HH and Park YS: Severity of nonalcoholic fatty liver disease on sonography and risk of coronary heart disease. J Clin Ultrasound 45: 391-399, 2017.

40. Livak KJ and Schmittgen TD: Analysis of relative gene expression data using real-time quantitative PCR and the 2(-Delta Delta C(T)) method. Methods 25: 402-408, 2001

41. Hong J, Kang B, Kim A, Hwang S, Ahn J, Lee S, Kim J, Park JH and Cheon DS: Development of a highly sensitive real-time one step RT-PCR combined complementary locked primer technology and conjugated minor groove binder probe. Virol J 8: 330,2011

42. Zhang Q, Zhao Y, Zhang DB and Sun LJ: Effect of Sinai san decoction on the development of non-alcoholic steatohepatitis in rats. World J Gastroenterol 11: 1392-1395, 2005.

43. Brunt EM, Janney CG, Di Bisceglie AM, Neuschwander-Tetri BA and Bacon BR: Nonalcoholic steatohepatitis: A proposal for grading and staging the histological lesions. Am J Gastroenterol 94: 2467-2474, 1999.

44. Haukeland JW, Damås JK, Konopski Z, Løberg EM, Haaland T, Goverud I, Torjesen PA, Birkeland K, Bjøro K and Aukrust P: Systemic inflammation in nonalcoholic fatty liver disease is characterized by elevated levels of CCL2. J Hepatol 44: 1167-1174, 2006.

45. Cynis H, Kehlen A, Haegele M, Hoffmann T, Heiser U, Fujii M, Shibazaki Y, Yoneyama H, Schilling S and Demuth HU: Inhibition of Glutaminyl Cyclases alleviates CCL2-mediated inflammation of non-alcoholic fatty liver disease in mice. Int $\mathrm{J}$ Exp Pathol 94: 217-225, 2013

46. Kirovski G, Gäbele E, Dorn C, Moleda L, Niessen C, Weiss TS, Wobser H, Schacherer D, Buechler C, Wasmuth HE and Hellerbrand C: Hepatic steatosis causes induction of the chemokine RANTES in the absence of significant hepatic inflammation. Int J Clin Exp Pathol 3: 675-680, 2010.

47. Shimizu Y, Murata H, Kashii Y, Hirano K, Kunitani H, Higuchi K and Watanabe A: CC-chemokine receptor 6 and its ligand macrophage inflammatory protein 3 alpha might be involved in the amplification of local necroinflammatory response in the liver. Hepatology 34: 311-319, 2001.

48. Chen W, Wang X, Huang LI and Liu BO: Hepcidin in non-alcoholic fatty liver disease regulated by the TLR4/NF- $\mathrm{B}$ signaling pathway. Exp Ther Med 11: 73-76, 2016.

49. Hassan K, Bhalla V, El Regal ME and A-kader HH: Nonalcoholic fatty liver disease: A comprehensive review of a growing epidemic. World J Gastroenterol 20: 12082-12101, 2014.

50. Zhang J, Tan Y, Yao F and Zhang Q: Polydatin alleviates non-alcoholic fatty liver disease in rats by inhibiting the expression of TNF- $\alpha$ and SREBP-1c. Mol Med Rep 6: 815-820, 2012.

51. Long YC and Zierath JR: AMP-activated protein kinase signaling in metabolic regulation. J Clin Invest 116: 1776-1783, 2006.

52. Kohjima M, Higuchi N, Kato M, Kotoh K, Yoshimoto T, Fujino T, Yada M, Yada R, Harada N, Enjoji M, et al: SREBP-1c, regulated by the insulin and AMPK signaling pathways, plays a role in nonalcoholic fatty liver disease. Int J Mol Med 21: 507-511, 2008. 